\title{
Téoros
}

Revue de recherche en tourisme

\section{Le tourisme d'agrément à Montréal : une clientèle en déclin ?}

\section{Chantal Langelier}

Volume 10, numéro 3, novembre 1991

Destination Montréal : d’hier à demain

URI : https://id.erudit.org/iderudit/1079201ar

DOI : https://doi.org/10.7202/1079201ar

Aller au sommaire du numéro

Éditeur(s)

Université du Québec à Montréal

ISSN

0712-8657 (imprimé)

1923-2705 (numérique)

Découvrir la revue

Citer cet article

Langelier, C. (1991). Le tourisme d'agrément à Montréal : une clientèle en déclin ? Téoros, 10(3), 35-39. https://doi.org/10.7202/1079201ar d'utilisation que vous pouvez consulter en ligne.

https://apropos.erudit.org/fr/usagers/politique-dutilisation/ 


\section{Le tourisme d'agrément à Montréal: une clientèle en déclin?}

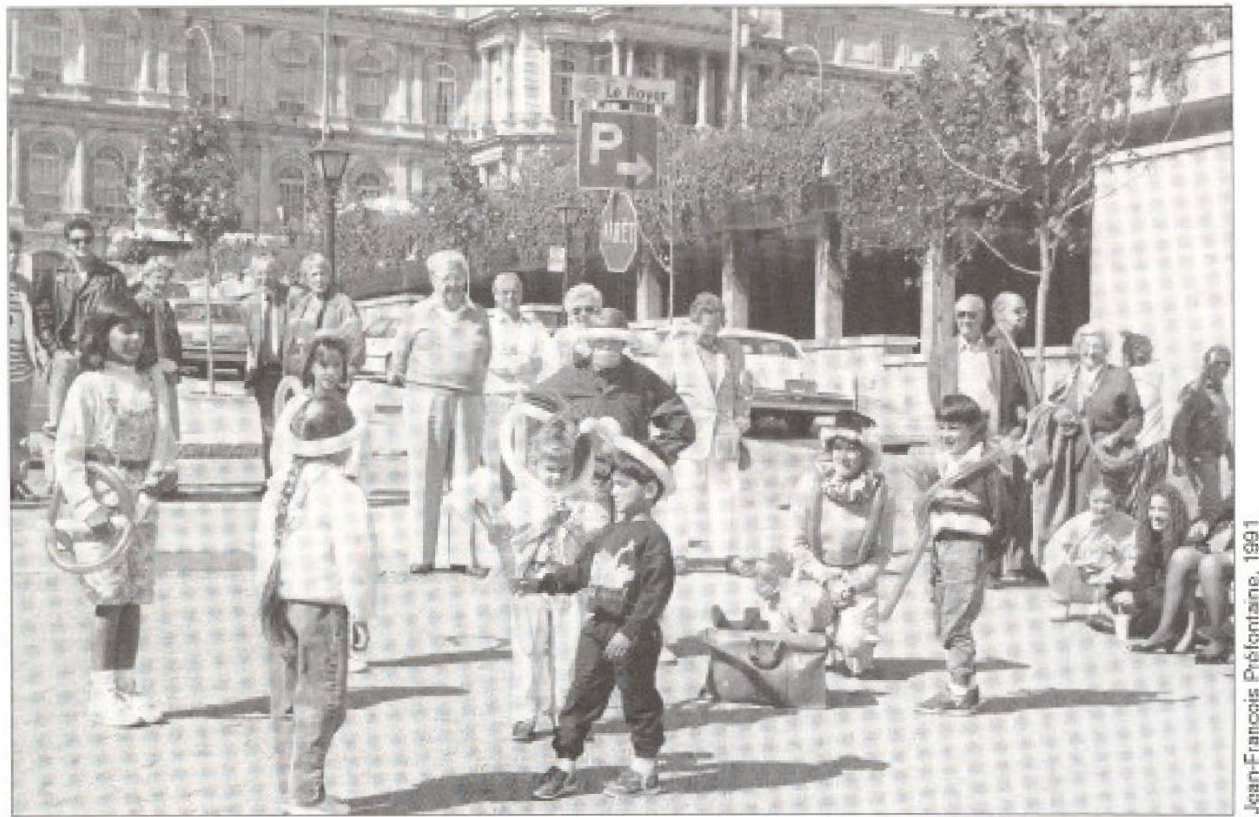

Les habiudes de wyages des fouristes changen el préreniement, les vacances en fanille ont wa regain de popularite.

\section{Le tourisme d'agrément:} une définition s'impose...

La Conférence internationale sur les statistiques des voyages et du tourisme, tenue conjointement par l'Organisation mondiale du tourisme et Tourisme Canada en juin dernier, a permis à des professionnels de plus de 90 pays de s'entendre sur la définition des termes touristiques, ainsi que sur un système de classification des activités du tourisme. Les résultats de ce consensus seront soumis pour approbation à la Commission de statistique des Nations Unies en février 1993, mais on observe déjà une nette volonté de ralliement autour de ces définitions de la part des intervenants touristiques internationaux.

Notre préoccupation actuelle concerne le motif de la visite qu'Antonio Merediz Montero a clairement défini lors de cette conférence: "Le motif de la visite n'est autre que la raison pour laquelle le visiteur prend la décision d'entreprendre le voyages (1),

Madame Chantal Langelier est directrice-adjointe de la recherche a l'office des congres et du tourisme du Grand Montréal IOCTGMB.
La classification des visiteurs par motif de visite a fait l'objet d'une recommandation de la part des participants de la conférence d'Ottawa. Il a été proposé que le motif principal de visite soit segmenté en trois catégories: le voyage d'agrément, les motifs professionnelsetles autres motifsà caractère touristique ${ }^{(2)}$, tels que proposés par Montero. Selon sa classification, les visiteurs qui se déplacent pour des vacances, visites culturelles, pratiques de sports, visites à des parents ou autres types de voyages d'agrément font partie de la même division.

Les définitions en usage au Canada ressemblent à peu de choses près à cellesci, si ce n'est que Statistique Canada fait, des visites à desparents ou amis, un segment particulier non inclus dans les voyages d'agrément. Cette classification nous semble plus pertinente puisque la composante visite à des parents a un impact économique très minime pour la région visitée. Lastructure des définitions utilisées par Statistique Canada s'adapte micux à notre analyse dans la perspective où l'aspect économique du tourisme doit être pris en considération.

\section{Examen de l'évolution du tourisme d'agrément à Montréal}

Statistique Canada effectue périodiquement. deux types d'enquête auprès des voyageurs: une enquête sur les voyages internationaux et une sur les voyages des Canadiens. Entreprise pour la première fois en $1980 \mathrm{et} \mathrm{à}$ tous les deux ans depuis, l'enquête sur les voyages des Canadiens fournit, entre autres, des renseignements sur les motifs de voyage. Elle constitue une source de données récurrentes parfaitement comparables et ce, pour les 10 dernières années. L'enquête sur les voyages internationaux fut, pour sa part, menée une première fois en 1980 puis reprise en 1990 et pourrait certainement fournir un portrait comparatif intêressant, mais les résultats de l'enquête de 1990 pour la région métropolitaine de recensement de Montréal ne sont pasencore disponibles au moment de la rédaction de cette analyse.

Nous utiliserons done comme base comparative, l'enquête de Statistique Canada sur les voyages des Canadiens, de 1980 à 1990. La population de cette étude couvre près de $65 \%$ de la clientèle touristique montréalaise, ce qui forme un échantillon assez appréciable pour fournir un portrait général de l'évolution đu segment tourisme d'agrément à Montréal.

Le tableau 1, ci-dessous présente une analyse de tendances des divers segments de touristes canadiens en visite à Montréal:

Selon cette analyse de tendances, le tourisme d'agrément constitue une clientèle en constante diminution à Montréal, Les chiffres: de $22 \%$ au début des années 1980 , les touristes canadiens dont le motif principal de voyage était l'agrếment ne représentent plus que $17 \%$ du marché touristique montréalais en 1990, soit une perte de 5 points pour une diminution réelle de $14 \%$ en 10 ans.

A l'ère où les voyages font de plus en plus partie du mode de vie des Nord-Américains, ces résultats peuvent sembler très surprenants... Doit-on attribuer cette baisse 
des voyages d'agrément à un problème d'image auprès des Canadiens et des Québécois, ou assistons-nous à une modification généralisce des habitudes de voyage des Nord-Américains?

La validité de l'hypothèse de perception négative de Montréal en tant que destination peut être remise en cause puisque le nombre de Canadiens en voyage d'affaires ou en congrès à Montrẹ́al a affichéc une croissance positive depuis la dernière décennie. En effet, ce segment qui comptait pour $26 \%$ en 1980 , se chiffre maintenant à $29 \%$. Ce gain de 3 points se traduit en une croissance effective de $17 \%$ au cours des 10 dernières années pour la clientèle canadienne des voyages d'affaires. La seconde hypothèse semble donc, à première vue, plus plausible: les comportements de voyage des Nord-Américains évoluent...

\section{Une tendance nord-américaine}

L'Institut canadien de recherche sur le tourisme (ICRT) qualifiait les années 1980 de décennie des voyages d'affaires et affirmait que: wes voyages d'affaires au Canada durant les années 1980 ont joui d'une croissance remarquable par rapport aux voyages d'agrément et aux visites de parents et amis $w^{(3)}$.

En effet, selon l'enquête sur les voyages des Canadiens, le segment des voyages d'affaires au Canadaa affiché une croissance de $30 \%$ entre 1980 et 1990 , accroissant la part de marché de cette composante de $12.5 \%$ en 1980 a $16 \%$ en 1990 . Les voyages d'agrément des Canadiens comptaient pour $45 \%$ du marché des voyages internes en 1980. Ils n'occupent plus que $39 \%$ du marché en 1990 , soit une perte de $6 \%$ de part de marché en dix ans.

Le nombre de Canadiens qui voyagent par agrément a diminué de $9 \%$ au cours de la dernière décennie. Faut-il rappeler que le pays connaît sa deuxième récession économique en dix ans? Laurie McDougall soulignait, dans un article paru dans Infovoyages, que le segment des voyages d'agrément est cèlui qui a êté le plus affecté lors de la crise économique de 1982: «Au cours de la dernière récession (1982), ce sont les voyages d'agrément qui ont réagi le plus rapidement et le plus vivement. Les Canadiens ont effectué $14 \%$ moins de voyages de ce genreen 1982 qu'en $1980 w^{(4)}$.

GRAPHIQUE 1

Évolution des segments de touristes canadiens en visite à Montréal de 1980 à 1990

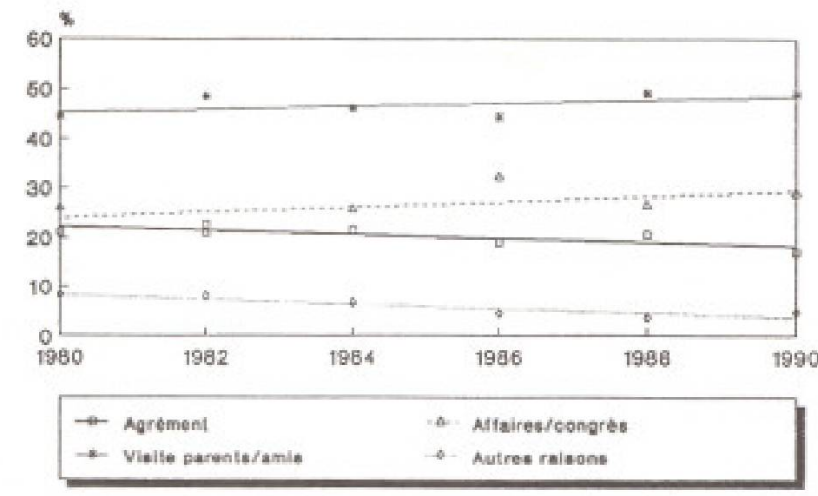

Sournt ENC, Btatietique Canada.

TABLEAU 1

Évolution des motifs de voyage des touristes Canadiens entre 1980 et 1990

\begin{tabular}{|c|c|c|c|c|c|}
\hline \multirow[b]{2}{*}{ Motif } & \multirow[b]{2}{*}{1980} & \multirow[b]{2}{*}{1990} & \multirow{2}{*}{$\begin{array}{r}\text { Variation } \\
1990 / 1980 \\
(000)\end{array}$} & \multicolumn{2}{|c|}{ Part } \\
\hline & & & & $\begin{array}{r}1980 \\
\%\end{array}$ & $\begin{array}{r}1990 \\
\%\end{array}$ \\
\hline Affaires & 9351 & 12168 & 30.1 & 12.5 & 16 \\
\hline $\begin{array}{l}\text { Agrément } \\
\text { Raisons }\end{array}$ & 33279 & 30405 & -8.6 & 45 & 39 \\
\hline $\begin{array}{l}\text { personnelles } \\
\text { Visite aे des }\end{array}$ & 5108 & 4923 & -3.6 & 7 & 6 \\
\hline parents & 26749 & 30830 & 15.3 & 36 & 39 \\
\hline $\begin{array}{l}\text { Total voyages } \\
\text { d'une nuit ou } \\
\text { plus }\end{array}$ & 74487 & 78326 & 5.2 & 100 & 100 \\
\hline
\end{tabular}

La rationalisation des dépenses des ménages provoquée par les récessions est une explication plausible de la croissance des visites à des parents ou amis au cours des dix dernières années. Le séjour chez des parents ayant des répercussions moindres sur le portefeuille familial qu'un séjour à l'hôttel!

Il est encore un peu tôt en 1991 pour pouvoir faire le bilan de la crise économique actuelle par contre, plusieurs signes nous portent à croire que les tendances seront généralement les mêmes qu'en 1982. Comme aprè̀s toute période de récession, une reprise devrait se faire ressentir des 1992. C'est ce que démontrent les prévisions économiques pour l'industrie touris- tique de l'Institut canadien de recherche en tourisme reproduites au graphique 2 .

Preuve est faite que Montréal n'est pas seule à assister à un déclin de sa clientè̀le d'agrément puisque l'ensemble du Canada affiche les mêmes tendances mais, qu'en est-il des États-Unis?

Dans un article paru dans la revue Téoros de juillet 1990, Elizabeth Stewart du U.S. Travel Data Center rapporte, en parlant de nos voisins du Sud, que: uDe 1979 à 1984 , les voyages d'agrément ont affiché des baisses annuelles moyennes d'à peu près $4 \%$. Malgré une reprise au cours des cinq derniêres années de la décennie quand leur nombre a augmenté de $5 \%$ par année, les 

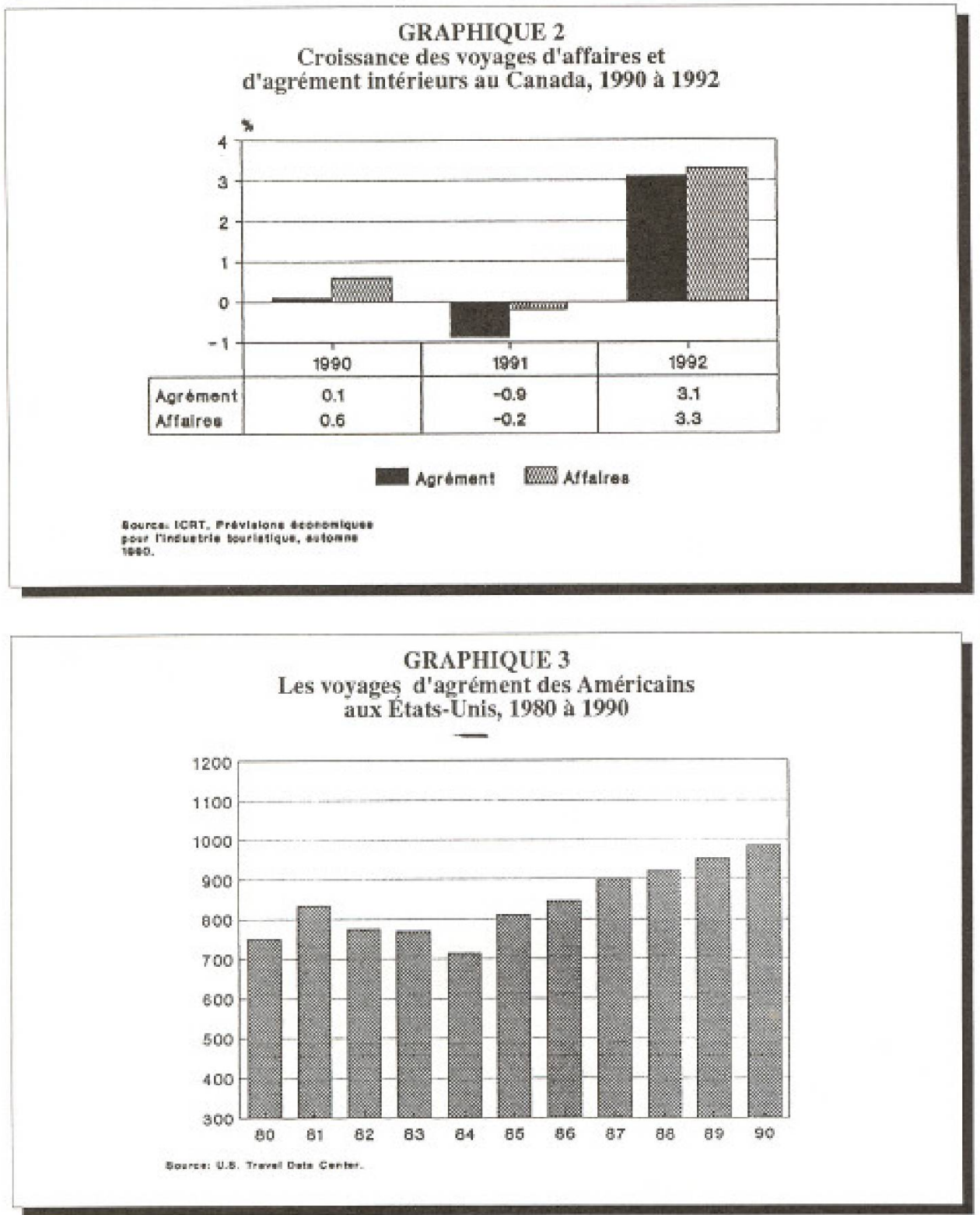

voyages d'agrément ont crû de moins d'un pour cent par annếe dans l'ensemble sur toute la décennie ${ }^{\text {s }}$.

Les voyages d'agrément des résidents des États-Unis ont tout de même affiché une hausse de plus de $30 \%$ entre 1980 et $1990 .$. Un net écart par rapport aux Canadiens pour qui ces voyages ont diminué de prês de $10 \%$ au cours de la même période. Il faut toutefois préciser que les statistiquessur les voyages d'agrément du U.S. Travel Data Center incluent les visites à des parents ou amis qui comptent pour $46 \%$ des voyages d'agrément. Si l'on utilise la même classification quecelle qui esten usage aux ÉtatsUnis, les résultats seraient un peu diffétents: la combinaison des voyages d'agrément avec les visites à des parents et amis au une renaissance du tourisme et (...) les années 1970 par une sorte de ressaisissement. Depuis les débuts des années $1980^{\circ}$ on semble s'acheminer vers une décennie troisième vague oủ les habitudes changent substantiellement. Peut-on prophétiser en supposant que les années 1990 favoriseront une seconde renaissance du tourisme ${ }^{(6)}$.

Monsieur Demers avait-il un don de clairvoyance ou était-ce sa compréhension globale de l'industrie touristique qui déjà en 1985, s'avérait juste? Quoi qu'il en soit, nous ne pourrons confirmer ses dires que dans quelques années. Toutefois, nous retenons que le tourisme est en constante evolution, en proie à des changements de comportement globaux de société.

Une étude effectuée en 1989 par la firme américaine Yankelovich, Clancy, Schulman faisait état des modifications des habitudes de consommation des Américains, modifications qui s'appliquent aussi aux Canadiens mais dont les effets seront retardés par rapport aux États-Unis. JeanMarc Bissonnette résumait dans un article paru dans la revue Téoros de juillet 1990 , les points saillants de cette étude: $\propto(. .$.$) on$ peut dégager un certain nombre de tendances chez nos voisins américains, pour la prochaine décennie, qui toucheront les modes de consommation touristique. Il $\mathrm{s}$ "agit du vieillissement de la population, $\mathrm{du}$ repli sur soi (cocooning), de la rationalisation, de l'accroissement du temps de loisir de lapriorité accordée à la qualité du service et à la responsabilité corporative et de la tendance qui veut que les riches seront de plus en plus riches ${ }^{\circ}$.

Les annees 1990 devront tenir compte de la clientèle des baby boomers maintenant au début de la quarantaine, mais qui se retirera du marché du travail dans quelques années. Ce créneau aura suffisamment d'argent et de temps à consacrer à de longues vacances. Ce sont eux qui aujourd hui ont fondé une famille et préfêrent se reposer à la maison afin d'amenuiser la pression quotidienne du travail et de la vie en société. Ce phếnomène de repli sur soi (cocooning) fait suite à un essoufflement des familles où à la fois l'homme et la femme sont présents sur le marché du travail et toù les tâches domestiques constituent un fardeau supplémentaire aux exigences de la vie quotidienne. Le cocooning explique en partie la décroissance du tourisme d'agrément au Canada. 
GRAPHIQUE 4

Les comportements de woyage des "baby boomers"

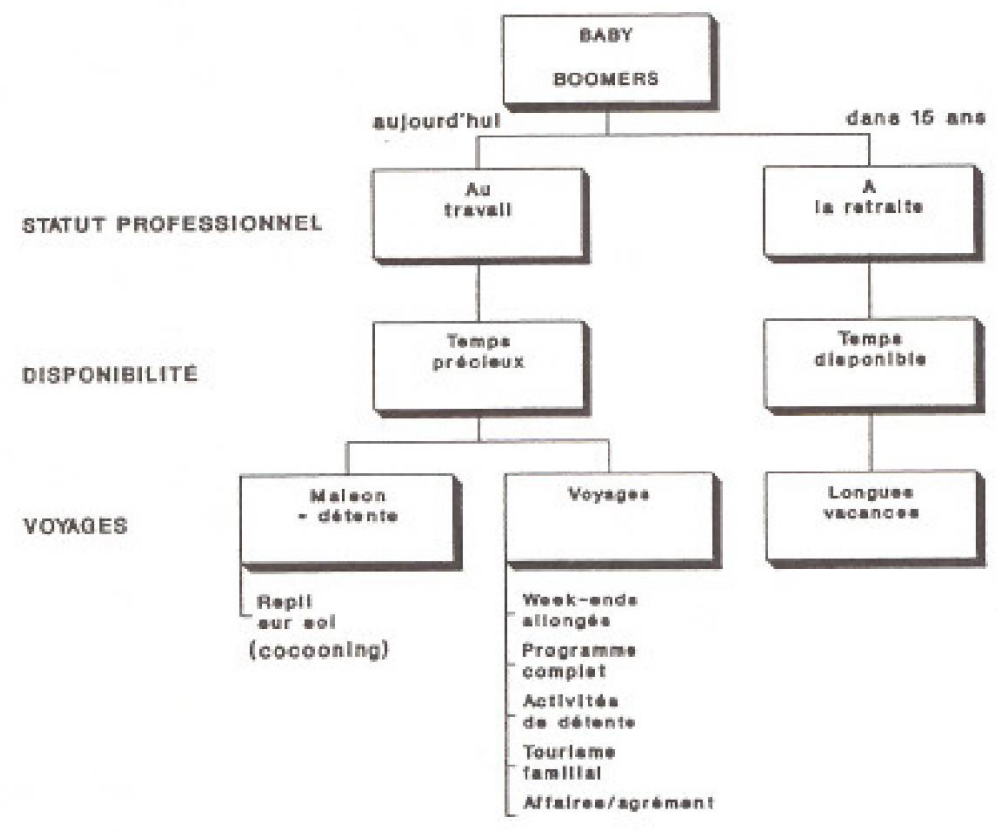

Le temps disponible pour effectuer des voyages constitue aussi un facteur important de la modification des habitudes de voyage. Les longs week-ends remplacent de plus en plus les vacances annuelles, ce qui implique que les distances parcourues doivent nécessairement être plus courtes. Explication plausible au fait que les ÉtatsUnis affichent une croissance des voyages d'agrément, les Américains préférant voyager chez eux. Ce comportement se fait nécessairement ressentir dans la balance touristique canadienne.

Les DINKS (Double Income No Kids) des annés 1980 ont maintenant des enfants et on observe un accroissement de la demande pour le tourisme familial et les activités de détente. Ayant peu de temps à consacrer à la préparation du voyage, ceux-ci préferent demander à une agence de woyages de s'en occuper. La rationalisation du temps des ubaby boomers; est aussi à l'origine d'une nouvelle tendance: la combinaison des voyages d'affaires et d'agrément. Dans un article écrit pour un congrès du Travel and Tourism Research Association en 1990, Cam Davis de l'ICRT relevait l'importance grandissante du phénomène de fusion des voyages d'agrément avec les voyages d'affaires: *CRTI, in its 1989 Business Traveller Survey, found 41 per cent had combined a business and pleasure trip at least once in $1989 \mathrm{~m}^{(\mathrm{w})}$,

Ces données s'appliquent au Canada mais, comme le souligne monsieur Davis, elles sont aussi vraies chez nos voisins américains si l'on se fie à une étude menée par le U.S. Travel Data Center: «(...) 45 per cent of business travellers combined business with vacation on at least one business trip throughout the years"

Et pour démontrer l'importance de ce nouveau segment en croissance, il ajoute: \&(...) half the business tripsincluded a vacation time period that equalled the business portion (119). $^{\text {(19) }}$.

Dans la moitié des cas, l'ajout du voyage d'agrément aurait done un impact aussi marqué que le voyage d'affaires auquel il se greffe.

\section{Et Montréal?}

La clientèle du tourisme d'agrément estelle réellement en déclin à Montréal? $\mathrm{A}$ cette question nous répondrons oui, mais...! Car il y a plusieurs points qui doivent être pris en considerration:

- Montréal n'est pas une exception puisque l'ensemble du Canada subit. les mểmes modifications de com= portements de voyage.

- Le tourisme d'agrếment est présentement en baisse à Montrétal mais la croissance de ce segment devrait reprendre dès 1992, tout comme partout ailleurs au Canada.

- Les deux récessions connues au cours des dix dernières années ont peut-être eu pour effet de déplacer une partie de la clientèle d'agrément vers le segment de visite à des parents ou amis.

- Les statistiques sur les voyages dont le motif principal est les affaires cachent une foule de données précieuses masquant l'importance du tourisme d'agrément greffé au voyage d'affaires... La segmentation des marchés est peut-être trop nette entre le segment des affaires et celui de l'agrément lors d'enquêtes telles que celles tenues par Statistique Canada. II faudrait réfléchir sur la possibilité d'ajouter un nouveau motif principal de voyage: la combinaison voyage d'affaires et d'agrement. Un tel ajout permettrait certainement de mieux cerner l'impact de cette nouvelle tendance.

La décroissance du tourisme d'agrément ne peut être qualifíe de problème montréalais puisque le phếnomène s'observe à l'échelle canadienne. Plusieurs facteurs. sont à l'origine de cette diminution, la plupart étant reliés à une modification du comportement social d'une majorité au sein de la population nord-américaine, soit les baby boomers. L'industrie touristique montréalaise $n^{\dagger}$ a d'autres choix que de tenir compte des besoins de cette génération si elle veut se positionner face à la concurrence féroce des autres villes nord-américaines. Le développement de produits adaptés aux voyages d'affaires-agrément constitue une option que Montréal devrait sérieusement envisager. A cet effet, le directeur de l'Institut canadien de recherche sur le tourisme, David Redekop, écrivait en juin dernier que le marché du voyage d'affairesagrément constitue: $\propto(. .$.$) l'un des marchés$ touristiques de croissance en Amérique du Nord dans les années 1990. (...) ce marché esten plein essor et (que) les personnes sont disposées à opter pour des vacances de qualité (thêâtre, vie nocturne, magasinage de luxe) ${ }^{\text {(11)? }}$. 
L'exploitation du créneau des vacances familiales constitue aussi une option non négligeable pour augmenter la part des voyages d’agrément dans le marché touristique montréalais. Il va sans dire que de multiples solutionspeuvent êtreenvisagées pour faire valoir Montréal auprès du touriste d'agrément. Par contre, le repositionnement de Montréal doit se faire rapidement car l'actuelle demande pour des produits touristiques familiaux se poursuivra dans les années 1990 , alors que le tourisme du dểbut đu siècle prochain sera plutôt axé vers une clientèle à la retraite. L'occasion est bonne pour Montréal, il s'agit de la saisir avant que les principales villes concurrentes aient pris une longueur d'avance!

\section{Notes et références}

(1) Antonio Merediz Montero, Statistiques touristiques - definitions et classifications de base. document de travail présenté aे la Conférence internationale sur les statistiques des voyages et du tourisme, 25 juin 1991, Ottawa, Canada, p. 22

(2) Conlerence internationale sur les statistiques des worages et du tourisme. Russolutions de la Confurence d’Ottawa, 28 juin 1991, p. 7.

(3) Institut canadien de recherche sur le tourisme. Perspectives sur les voyages d'affaires - points saillants, mars $1990, p, 1$,

14) Laurie McDougall, "Les récessions, le passé et le présente, dans Info-voyages, no 87-003, hiver 1991, p. 3

(5) Elizabeth Stewart, a Les vovages des Americains aux États-Unis.., dans Táoros, vot. 9, no 2. julliet 1990, p. 9.

(6) Jacques Demers, «Nos touristes depuis 20 ans dans Téoros, vol. 4, no 2, juillet 1985, p. 5.

(7) Jean-Marc Bissonnette, *Indicateurs du changement de la onsommation touristicue des Amé ricains ", dans Téoros, wol. 9, no 2, juillet 1990, p. 19

(8) Cam Davis, «Capturing the Business/Pleasure Market , dans Tourism Research: Meeting the Needs of Industry. Conterence Proceedings. TTRA Canada 1990, p. 43.

(9) Idem.

(10) idem.

111) David Redekop, Les résidents de la région du Pacificue aux Ėtats-Unis mettent le cap sur to Canada, Institut canadien de recherche sur le tourisme, juin 1991, p. 2.

\section{PROCHAIN COLLOQUE TÉOROS}

Comme chaque année depuis 4 ans maintenant, la revue Téoros tiendra un colloque d'une journée dans le cadre du prochain congrès de l'ACFAS (Association canadienne française pour l'avancement des sciences). Il aura lieu le 13 mai 1992 à 1'Université de Montréal sous le thème: Le tourisme et la ville. Les nombreuses facettes du tourisme urbain seront analysés par six études de cas portant sur autant de grandes villes d'ici et d'ailleurs. Réservez la date du colloque; le prochain numéro de Téoros vous fera connaître le programme détaillé et les conférenciers.

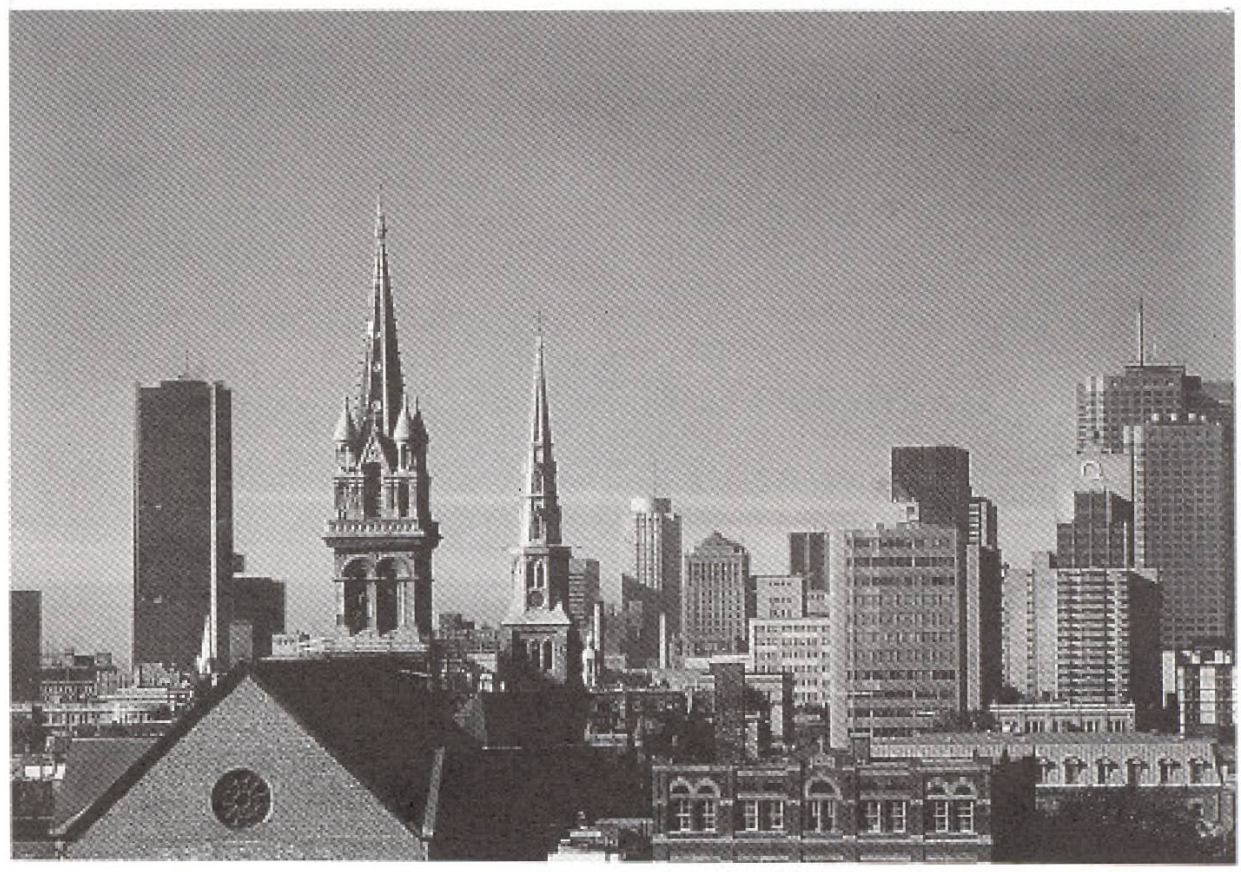

